\title{
The Impact of Memory Load on Customer Choice Behavior
}

\author{
Hao-Te Lu, Tso-Jen Chen, Yi-Chou Wang* \\ College of Management, Tainan University of Technology, Tainan, Taiwan \\ Email: t90063@mail.tut.edu.tw
}

Received July 2014

\begin{abstract}
There are only limited things that human brain can process at the same time, and people are adapted to save mental resources for things that are more important. Earlier research has shown that heuristics is employed to lighten brain load when people have to make quick decisions. However, it is not clear whether people will make rational choices if they have plenty of time. The purpose of this study is to investigate whether mental resources more or less will affect consumer behavior. In the experiment feeling/thinking (intuition vs. rational), working memory load (numbers memorization), will be employed to understand participants' changing behaviors.
\end{abstract}

\section{Keywords}

Memory Load, Choice Behavior

\section{Introduction}

Previous studies assume that people are inherently rational; in other words, people become irrational only in certain situations, such as under pressure, mental overloading, time effect. Yet when human beings have sufficient time or even too much time for consideration, will they ultimately have rational consumer behaviors? Even researchers have observed the change of consumer behaviors; they still cannot determine whether the change of such behaviors was caused by heuristic method effect or other factors. Several laboratory studies have shown that human subjects do exercise and have diet in order to get good shape, but they still want to have some cake as rewards [1]. When people want to have ice cream, the choice was made fast. However, if longer time was given, the number of subjects who choice ice cream decreased [2].

Although previous researches have considered issues such as reason, intuition, circumstances, most of the studies focused on pressure effects. It is because time pressure is relatively easier to generate during the tests, and the effects are more obvious. We hope to explain how specific circumstances affect the decision-making behaviors by performing several laboratory experiments. Subjects were categorized into a control group (stimulus without memory loading) and experimental groups (stimulus receiving memory loading), using convenience and random sampling methods. Convenience sampling regards nonprobability sampling, which involves samples drawn from a part of population that is easy to reach. Each group was selected by similar population in order to reduce bias. After the experiment, the impacts on subjects due to the effects of an independent variable

${ }^{*}$ Corresponding author. 
were observed. The purposes of this research are to understand: stimulus-response circumstances \& memory loading circumstances.

\section{Literature Review}

\subsection{Decision-Making Process}

What is a decision? A decision is a choice between two or more alternatives (Webster's 9th Dictionary). Decision means that people try to come up with a solution to resolve uncertainty or disputes. We all make various decisions in our day-to-day life. Ironically, people make more poor decisions than they think. What is Decision Making? Stoner and Freeman [3] define decision making as a process of identifying and selecting a course of act. Wood et al. [4] define that decision making lies at the heart of a successful organization and as such it is important to consider different decision making processes.

Shafir and Tversky [5] define decision making: who has well defined preferences that do not depend on a particular description of the options or on the specific methods for eliciting preference. Shafir \& Tversky [5] indicate that the ideal decision maker who makes decisions based completely on logic and whose decision is not changed based on the way the choices are presented.

Decision making is a process of identifying and choosing alternatives based on values and preferences. It is the process of reducing uncertainty about alternative choices to be made. If there is no uncertainty, you do not have a decision. If you only have alternatives, you do not have a decision. When facing multiple objects in a short time, people are making decisions. Yet if they do not have sufficient information and knowledge (ability), it may cause conflicts and distress, which leads to indecisions or wrong decisions. Making a decision implies probability of success and fits with goals, desires, and lifestyle.

Osland, Kolb \& Rubin [6] posits that understanding the problem, defining the problem, pointing out decisionmaking goals, setting decision criteria, assigning priority for each decision-making criterion, developing multiple possible options, choosing the best possible options for implementation, evaluating the decisions represent the rational decision-making process. A decision involves pursuing the best goal or objectives, and so goals and objectives come before decision making.

\subsection{Rational Decision-Making}

Gulick \& Urwick [7] propose the Rational Comprehensive Model by holding that rational decision-makers can use knowledge and resources to evaluate various pros and cons then make decisions. It is a logical way to analyze decision-making, and decision-making process is simplified and sought to maximize the benefit. All possible options to solving problem are identified and the costs and benefits of each option are assessed. It means decision marker will choose the option that brings the greatest benefit even though it will be costly in time and mental resources and might be difficult to identify.

Robbins \& Timothy [8] argues that decision making follows a process or orderly path from problem to solution. These steps include recognizing problems, setting objective assessment criteria, defining the best solution, and starting primary analysis and implementation. Rational decisions seek to optimize the beliefs of the decision maker, and rational decisions should be logical and objective, unbiased, and based on facts. Good decision making, while not representative of good results, has a higher probability to achieve the goals desired. Based on the assumptions of rational decision theories, all decisions should get the same result if there is enough information and ability for the decision to be made. In fact, in most cases, it is not true. It is because the limitations on human's capabilities, such as lack of information, knowledge, time, etc., will change the result.

\subsection{Bounded Rationality}

Even though people in the management field always place an emphasis on analysis and rational decision-making, a number of studies have pointed out that most of the decision-making behaviors are affected by personality, mental state and political considerations [9]. Since humans cannot digest and understand all the information, we will simplify the problem and only look for the most important factor. It is because our decision-making process is too often cut short by the incomplete information we have; therefore, we can only come up with the most obvious solution. We will only seek a satisfactory solution, not the optimal one. In real life, psychological factors often play roles in people's decision-making behavior and thus should be added as a reference for decision 
analysis.

Human has limited cognitive abilities and knowledge and, therefore, cannot have all the information to get the desirable result. People meet his/her expectations of decision-making, and this decision-making behavior is a limited rational behavior (Bounded Rationality) instead of the optimal solution. It also represents an acceptable choice that can be done. In structured situations, decisions involve two components: environmental demands the individual incentives (positive or negative) [10].

Traditional research often assume rational and objective as the major decision-making behavior. In fact, human beings often ruin rational behavior because of personal reasons or being emotional. When information is insufficient or asymmetric, people who lack of knowledge cannot make effective interoperation and conclusion, resulting in incomplete rational behaviors. And consequently the irrational behaviors cause bias that facilitates additional non-rational behaviors.

\subsection{Cognitive Load \& Memory}

Cognition is using concepts, perception, imagination and other mental activities to determine or to obtain knowledge of the process. The processes of thinking and evaluation which can be defined to individual cognitive system perform a specific work and result of loading [11].

Cognitive load refers to cognitive resources are focused and used during learning and problem solving [12], cognitive load is that learners have a working memory with limited capacity when dealing with new information [11], which involves information can be retained in short-term memory. Short-term memory is limited capacity, but enormous amount of information stored in long-term memory. The working memory is limited in capacity and duration, can hold only a limited number of items at a time. If there are more than ten information in one possesses overload of working memory may occur.

In this study, Memory Load is represented by the brain's short-term memory. Short-term memory capacity is small, most of the papers claimed it is seven \pm two units. The magical number seven, plus or minus two: Some limits on our capacity for processing information [13].

Memory-based choices tend to reflect relatively more intuitional process, whereas stimulus-based choices reflect relatively more rational process [14]. Thus, memory-based choices are tend to immediately reaction and stimulus-based choices are more cautious. In memory load situation, consumers need to maintain the memory task; in contrast to stimulate base (without any mental load conditions), consumers do not need to maintain these working memories and can respond with adequate mental resources.

As mentioned above, intuitive is used for decision-making in order to reduce brain loads while people who use stimulus-based responses tend to be more cautious. This is because the experimental subjects are working on task confirmation, while working to maintain the resources in the work of memory. In a converse manner, memory load processes do not require recalls any information and no working memory are consumed. Memory-based choice will reduce the cognitive load to tend choice of happiness is hedonistic side, but stimulate-base more support for choice of the appropriate choice; it is easy to guide the choice of basis in partial reason [14].

Ravi \& Itamar [15] suggest that consumers face uncertainty circumstance, they will prefer to choose the option which is reduce risk and contradictions; if allowed to increase the selected time (considering time) than original choose the option to avoid the risk (risk reduction), the number become smaller.

Obviously, memory loading would consume the brain resource compared with the stimulating situation. Memory loading will reduce the inference process, thus making rational thinking more difficult. Consequently, participants tend to choose easy way to compensate their hard work. Ravi and Klaus [16] published a literature on self-control emphasizes that temptation is costly. The temptation entails not only costs but also benefits for consumers. Self-signaling costs and benefits of temptation depend not only on the chosen item but also on the temptation from the non chosen options.

\section{Experimental Design}

Laboratory experiments is a research which exclude nonrelatives variables of impact factor in designed environment, the field experiment is to choose a real environment to carry out, the more realistic situation but poor handle of variables relatively. Experimental method is to make the individual change to observe the reaction of the experimenter to study the changes in the way. Although the experimental method is superior to traditional research methods, such as in Davis et al. [17] control capabilities, but does not replace traditional research, both 
have their own merits.

Because the field experiment may produce, sample space and the reality of differences, leading to the impact of external validity. Therefore researchers need to know how to do. In this study, use laboratory experiment research methods which can control various sources of variation and has best internal validity research method. Laboratory experiment by manipulation of experimental conditions can explore the nearly true behavioral response.

Only by allowing researchers to manipulate situations to study the problem can use laboratory experiments to. The laboratory experiment is trying minimizing the unpredictable and as possible as control all the variables. Experimental is manipulation one or more variables in a controlled environment, which also can verified relationship between variables. It is suitable for scientific research, description and control.

\section{Data Analysis and Discuss}

\subsection{Tasty and Healthy Food Experiment}

Sirgy [18] consumer researchers have explored product symbolism that possibly contributes to and changes the consumer's self-concept. This experiment chose food without brand. The experimental subjects (participants) were sophomore and junior students form Tainan University of Technology and Far-East University. In total, 74 male and 87 female participants completed the questionnaire with Likert 10 point scale. Table 1 depicted delicious and Health scale in this study.

In this experiment, it was found that pudding has the highest delicious index and yogurt has the lowest delicious index. Apple has the highest healthy index and chocolate cake has the lowest healthy index. According this delicious/healthy concept, this research was paired pudding with yogurt and chocolate cake with apple. This study assumed that students with healthy concept would choose apple and yogurt and that students with tasty concept will choose pudding and chocolate cake.

Experiment 1: Stimulus-based choices will tend to favor healthy food

This study modified experiment done by Rottenstreich [14] by showing desserts on the screen. Participants were asked to answer the following questions.

Hypothesis 1: Stimulus-based choice does not require brain loading. Therefore, participants tend to make decision rationally.

This research took 205 people for analysis. Computer measured results indicated that the shortest time to make a decision is 1.2 seconds and the longest time to make a decision is 8 seconds. The average time is $3.09 \pm$ 1.36 seconds. Interestingly, no matter what participants chose at the first, apple was the most chosen as the second choice. Although these experiment results do not support the hypothesis of healthy/tasty concept, this research used this data as control group.

Experiment 2:

Memory-Loading choices will tend to favor delicious food. This research modified experiment done by Rottenstreich et al. [14] by showing 10-digit telephone number on the screen. Participants were told to remember the number and reenter the memorized number to the computer after answering the following questions.

Hypothesis 2: Memory will increase the mental loading. Therefore, participants with mental loading tend to make decision intuitively. It means participants will choose chocolate cake more likely than apple that is healthier and preferred by the control group.

There were 195 people participated the experiment, 22 people typed the wrong 10-digits number to computer

Table 1. The Delicious and health scale.

\begin{tabular}{cccc}
\hline \multicolumn{4}{c}{ Delicious Index } \\
Pudding & Yogurt & Chocolate Cake & Apple \\
7.2 & 4.8 & 7.0 & 6.03 \\
& \multicolumn{2}{c}{ Healthy Index } & \\
Pudding & Yogurt & Chocolate Cake & Apple \\
5.3 & 6.8 & 4.2 & 8.16 \\
\hline
\end{tabular}


and were eliminated from the sample pool. Thus, this research only took 163 people for analysis. This study found that participants with mental loading by memorizing 10-digit number altered their choice making behavior by increasing the preference for delicious' food, which is consistent to previous research finding [14]. There was 77 participants choose apples and 86 participants chose chocolate cakes.

This study also found the in the experiment, the longest decision making time is 7.25 seconds and the shortest time is 1.35 seconds. The average time of choice making is $3.84 \pm 1.48$ seconds, which is significantly longer than the control group (Student T-test, ${ }^{* * *}, \mathrm{P}<0.001$ ) and suggests that choice making time is increased when people are with mental loading.

This study found that Chi-Square 7.955, Degrees of Freedom 1, P $=0.005$. The memory load participants expressed higher intuition behavior than stimulate participants. Therefore, this research proposes that participants with mental loading tend to make decision more intuitively. It is because people choose tasty foods intuitively. It means participants will choose chocolate cake more likely than apple that is healthier and rational compared by the control group.

\subsection{Results and Discuss}

In order to fill previous research gap, this study added the time-expanding factor along with three independent variables, including time, price and memory loading, which were proposed by Rottenstreich, Y., Sood, S., \& Brenner, L. [14], and Shiv, Baba and Alexander Fedorikhin [19]. Since memory loading would consume the brain resource which will make rational thinking more difficult, experiment participants tend to choose delicious food as a way to compensate their hard work. For instance, people who faced situation such as under extreme business pressure, working pressure, family issues, human relation problems and all kinds of high degree psychological stress need some other ways to release their pressure. When people do not have enough mental resource to resist temptation, they will intuitively select tasty food, such as fried chicken, barbecue and liquor without rational thinking.

\section{References}

[1] Schelling, T. C. (1984) Choice and Consequence. Harvard, University Press, Cambridge.

[2] Milkman, K.L., Rogers, T. and Bazerman, M.H. (2010) I'll Have the Ice Cream Soon and the Vegetables Later: A Study of Online Grocery Purchases and Order Lead Time. Marketing Letters, 21, 17-36. http://dx.doi.org/10.1007/s11002-009-9087-0

[3] Stoner, J.A.F. and Freeman, R E. (1989). Management. 4th Edition, Prentice-Hall, Inc., New Jersey.

[4] Wood, J., Wallace, J. and Zeffane, R.M. (2001) Organisational Behaviour: A Global Perspective. John Wiley \& Sons Australia Ltd., Australia.

[5] Shafir, E. and Tversky, A. (1995) Decision Making. In: Smith, E.E. and Osherson, D.N., Eds., An Invitation to Cognitive Science, 2th edition, MIT Press, MA.

[6] Osland, J.S., Kolb, D.A. and Rubin, I. M. (2001) The Organizational Behavior Reader. 7th Edition, Prentice Hall, Englewood Cliffs,

[7] Gulick, L. and Urwick, L. (1937) Papers on the Science of Administration. Institute of Public Administration, New York.

[8] Robbins, S.P. and Timothy, A.J. (2010) Organizational Behavior. 14th Edition, San Diego State University.

[9] Holloman, C. (1992) Using Both Head and Heart in Managerial Decision Making. Industrial Management, 34, 7-10.

[10] Simon, H.A. (1996) The Sciences of the Artificial. 3rd Edition, MIT Press, Cambridge.

[11] Sweller, J., Van Merriënboer, J.J.G. and Paas, F.G.W.C. (1998) Cognitive Architecture and Instructional Design. Educational Psychology Review, 10, 251-285. http://dx.doi.org/10.1023/A:1022193728205

[12] Chandler, P. and Sweller, J. (1991) Cognitive Load Theory and the Format of Instruction. Cognition \& Instruction, 8, 293-240. http://dx.doi.org/10.1207/s1532690xci0804_2

[13] Miller, G.A. (1956) The Magical Number Seven, Plus or Minus Two: Some Limits on Our Capacity for Processing Information. Psychological Review, 63, 343-355. http://dx.doi.org/10.1037/h0043158

[14] Rottenstreich, Y., Sood, S. and Brenner, L. (2007) Feeling and Thinking in Memory-Based versus Stimulus-Based Choices. Journal of Consumer Research, 33, 461-469. http://dx.doi.org/10.1086/510219

[15] Ravi, D. and Itamar, S. (2003) The Effect of Forced Choice on Choice. Journal of marketing research, 40, $146-160$. 
http://dx.doi.org/10.1509/jmkr.40.2.146.19229

[16] Ravi, D. and Klaus, W. (2012) Self-Signaling and the Costs and Benefits of Temptation in Consumer Choice. Journal of Marketing Research, 49, 15-25. http://dx.doi.org/10.1509/jmr.10.0490

[17] Davis, J.M., Bailey, S.P., Jackson, D.A., Stresner, A.B. and Morehouse, S.L. (1993) Effects of Serotonin (5-HT) Agonist during Prolonged Exercise to Fatigue in Humans. Medicine and Science in Sports and Exercise, 25, S78. http://dx.doi.org/10.1249/00005768-199305001-00440

[18] Sirgy, M.J. (1982) Self-Concept in Consumer Behavior: A Critical Review. Journal of Consumer Research, 9, 287300. http://dx.doi.org/10.1086/208924

[19] Shiv, B. and Fedorikhin, A. (1999) Heart and Mind in Conflict: The Interplay of Affect and Cognition in Consumer Decision Making. Journal of Consumer Research, 26, 278-292. http://dx.doi.org/10.1086/209563 